\title{
How can we improve the recognition, reporting and resolution of medical device-related incidents in hospitals? A qualitative study of physicians and registered nurses
}

\author{
Julie Polisena ${ }^{1,2^{*}}$, Anna Gagliardi ${ }^{3}$ and Tammy Clifford ${ }^{1,2}$
}

\begin{abstract}
Background: To explore factors that influence and to identify initiatives to improve the recognition, reporting and resolution of device-related incidents.

Methods: Semi-structured telephone interviews with 16 health professionals in two tertiary care hospitals were conducted. Purposive sampling was used to identify appropriate study participants. Transcribed interviews were read independently by one individual to identify, define and organize themes and verified by another reviewer.

Results: Themes related to incident recognition were the hospital staff's knowledge and professional experience, medical device performance and clinical manifestations of patients, while incident reporting was influenced by error severity, personal attitudes of clinicians, feedback received on the error reported. Physicians often discontinued using medical devices if they malfunctioned. Education and training and the implementation of registries were discussed as important initiatives to improve medical device surveillance in clinical practice.

Conclusions: Results from the telephone interviews suggest that multiple factors that influence participation in medical device surveillance activities are consistent with results for medical errors as reported in previous studies. The study results helped to propose a conceptual framework for a medical device surveillance system in a hospital context that would enhance patient safety and health care delivery.
\end{abstract}

\section{Background}

The US Food and Drug Administration (FDA) defines a medical device as an instrument used to diagnose, treat or prevent a disease or abnormal physical condition without any chemical action in the body [1]. Medical devices are used to monitor, replace or modify anatomy or physiological processes. They are important health care innovations, enabling effective diagnosis and treatment using less invasive techniques in many instances, and improving health care delivery and patient outcomes.

\footnotetext{
* Correspondence: Juliep@cadth.ca

${ }^{1}$ Canadian Agency for Drugs and Technologies in Health, 600-865 Carling Avenue, Ottawa, ON K1S 5S8, Canada

${ }^{2}$ Department of Epidemiology and Community Medicine, Faculty of Medicine, University of Ottawa, 451 Smyth Road, Ottawa, ON K1H 8 M5, Canada

Full list of author information is available at the end of the article
}

Despite their benefits, devices can be harmful. Incidents among medical devices have garnered widespread attention through frequent media reports of injuries, recalls, and class-action lawsuits. Such devices include catheters, infusion systems, surgical instruments, and implantable devices, including surgical mesh, pacemakers, stents, defibrillators, and artificial joints [2]. In a 2003 study of 65,826 incident base records, the rate of adverse events associated with medical device user-error incidents was 43.4 \% [3]. Post-market surveillance (PMS) used to collect data on adverse events associated with the use of medical devices enables us to address the risks of device-related adverse events sooner via the earlier detection that is facilitated by structured surveillance systems. This approach would prospectively monitor safety and effectiveness through its data collection without 
impeding access to innovative devices; more rapidly identify and communicate incident data to avoid further events; guide the development of training, organizational process improvement, or other patient safety interventions; direct decision-making about funding or replacement; and potentially inform the total product lifecycle by manufacturers.

We found three systematic reviews that identified factors influencing the frequency and reporting of incidents in a hospital setting have been conducted [4-6]. Lawton et al. developed a "contributory factors framework" from the published literature on factors that add to patient safety incidents in a hospital context. The authors found that two main contributory factors related to patient safety incidents were active failures (i.e., any failure in performance by the end-user) and individual factors (i.e., characteristics of the persona delivering the case that may contribute in some way to active failures) [4]. In addition, Pfeiffer et al. proposed a framework on barriers and motivators for incident reporting. They concluded that individual, organizational and incident reporting systems factors impacted reporting behaviour [6]. A systematic review identified 1676 factors contributing to patient safety incidents in 83 eligible studies, and categorized factors into 20 domains including active failure in performance or behaviour, clinician, team, institution, system, culture, training, accountability and patient factors [5]. Nonetheless, neither study was specific to devices and there was no investigation of factors related to incident recognition and resolution [4-6].

Polisena et al. conducted a systematic review on factors that influence device-related incident recognition, reporting and resolution of device-related incidents, as well as interventions or strategies to improve the recognition, reporting and resolution of device-related incidents. The authors expanded the scope to include other health technologies, such as drug therapies, diagnostic and screening tests, vaccines and surgical and non-surgical procedures, to ensure the comprehensiveness of the literature found. Among the 30 studies selected, most focused on factors that influence incident reporting [7].

The most effective medical device surveillance system that will improve patient safety in a hospital remains unknown. Further insight on how to design and implement surveillance systems at the hospital level is needed to more broadly and systematically prevent, and mitigate the impact of incidents. Our study objectives were to i) explore factors that influence device-related incident recognition, reporting and resolution and ii) describe barriers and existing and/or recommended initiatives to improve the recognition, reporting and resolution of device-related incidents with a particular focus on potential roles of physicians and nurses. For our study, resolution was defined as interventions used to reduce the risk of similar medical device-related incidents from reoccurring.

\section{Methods}

\section{Approach}

There is insufficient evidence in the published literature on the factors that influence physician's and nurse's roles in surveillance. A grounded qualitative approach was used to explore the perceptions, behaviours, practices, and experiences of physicians and nurses related to the use of devices. This approach elicits views and insights for a rich understanding of phenomena $[8,9]$. Physicians and registered nurses (RNs) were interviewed to explore factors that influence device incident recognition, reporting and resolution. The same interviews also solicited information about initiatives or strategies to improve the recognition, reporting and resolution of device-related incidents. The critical incident interviewing technique which asks respondents to provide detail about particularly relevant experiences also was employed [9]. Telephone interviews of approximately 30 mins were audio-recorded and transcribed verbatim (Additional file 1). The interviews were conducted between September and December 2013 in two Canadian teaching hospitals. A systematic review of the medical and grey literature on factors that influence common device incident and their recognition, reporting and resolution was supplemented by the results of our interview responses [7]. Ethics approval was received by the Ottawa Health Science Network Ethics Board and the University Health Network Ethics Board. Each study participant signed a consent form prior to the interview.

\section{Sample selection and recruitment}

Physicians and RNs at two tertiary care hospitals with over 1500 hospital beds combined, one in Ottawa and another in Toronto, Canada, were identified by research team members to collect information about their experiences with medical device-related incidents. The study sample varied by health profession sampling criteria. Potential study participants also were identified by the interviewees. To reduce the risk of skewed interview responses, we recruited six physicians from each hospital, who varied by specialization and, therefore, exposure to and use of different devices (two general surgeons, two orthopedic surgeons, two vascular surgeons, two cardiologists, two cardiac surgeons, two interventional radiologists). In addition, four RNs (two from each hospital) with experience in intensive care units or operating rooms (ORs) were recruited. Prospective interviewees were invited by electronic mail and asked to sign and return a signed consent form. Information from representative, rather than a large number of participants is needed in qualitative research. It is not meant to produce generalizable results but to provide an in-depth exploration of issues. Sampling was concurrent with data collection and analysis and was proceeded until no further unique themes emerged. Thematic saturation, where the categories were explained adequately and new 
categories do not emerge, was assessed upon review of codes derived based on responses for each interview question. If no new themes emerged related to factors that influence incident recognition, reporting and resolution, as well as recommendations to improve medical device surveillance, the authors deemed that additional interviews with physicians and RNs would not add new insights to the current responses [10]. As the interview questions were specific to medical device-related incidents in two teaching hospitals in the same jurisdiction, the authors deemed that thematic saturation was achieved after 16 interviews, where no new information was obtained and some redundancy was observed in the thematic categories in subsequent interviews.

\section{Data collection}

Data collection and analysis followed the principles of grounded qualitative research $[8,9]$. This method enabled the induction, or the drawing of ideas from information conveyed by participants, because it did not rigidly adhere to pre-existing constructs derived from established models or theories [11]. The critical incident technique was used to prompt interviewees to reflect upon significant or impactful events and provide a detailed description of the scenario. It has been used by others to explore medical equipment failures $[9,12]$. An interview guide was developed to ask users to describe how they recognized, reported and resolved a medical device-related incident and to provide suggestions or indicate initiatives underway to improve device surveillance in their local setting. Prior to commencing the interviews, the investigator ensured the interviewees' anonymity and confidentiality.

\section{Data analysis}

Qualitative analysis identified unique themes in an inductive manner $[13,14]$. Transcribed interviews were read independently by one individual (JP) to identify, define and organize themes and verified by another reviewer (ARG). A log was maintained of emerging codes, their definition, and a sample narrative illustrating the application of that code (open coding). The narrative was reviewed using the constant comparative technique to identify all instances of the coding framework, and to determine how to expand or merge thematic codes (axial coding). Two individuals (JP and ARG) compared their findings and achieved consensus through discussion. Coded text was tabulated by research objective to compare and interpret results, and MS Excel was used to organize the data. One reviewer (JP) reviewed the text repeatedly to ensure consistency in the interpretation of the transcripts. Major themes that emerged from the code schemes and patterns identified in the data helped to inform the development of a conceptual framework to improve the safety of medical devices in Canadian hospitals.

\section{Results}

\section{Characteristics of Interviewees}

Sixteen health care professionals, with years of practice that ranged between two and 39 years and from various specialities, were selected from two separate institutions to represent unique perspectives on medical device-related incidents.

\section{Medical device-related incident examples}

Types of medical devices commonly associated with incidents ranged from dialysis and extra corporeal life support machines and infusion pumps to implantable devices, such as catheters, stents, and inferior vena cava filters. Staplers also were mentioned by both clinicians and nurses as prone to incidents during a clinical procedure. One cardiologist responded that new medical devices were more prone to errors as a result of a learning curve associated with its use.

\section{Factors that influence recognition of medical device- related incidents}

Themes related to incident recognition included education and training, hospital staff knowledge and experience, performance of medical device, and warnings or advisories (Table 1). Exemplar quotes for each theme are available in Additional file 2: Table S1. Telephone responses revealed that the recognition of a medical device-related incident or malfunction is related to the clinician's past experience with the use and knowledge of device. Issues with medical devices also can be recognized if they did not operate according to the manufacturer instructions. Specific medical device features can alert the hospital staff when an error had occurred. Medical device performance was sometimes linked to their off-label use on patients with complex problems or an experimental treatment conducted. Warnings or notifications issued by manufacturers or regulators alerted the hospital staff to the increased risks on medical device malfunctions.

\section{Factors that influence reporting of medical device-related incidents}

Themes related to incident reporting include error reporting compliance, ethics, feedback on how reported information is used, information sharing, incentive for error reporting, institutional and professional cultures, and reporting system and process to the hospital, manufacturer, and regulator. It is not surprising that physicians did not want to work with medical devices that did not function as intended, especially if the incident was complex, and wanted to notify other potential users about their safety issues to reduce the risk of incidents with the specific device.

Numerous respondents felt that they were either encouraged or required to report incidents, malfunctions, 
Table 1 Thematic summary of interview responses

\begin{tabular}{|c|c|}
\hline Medical Device Surveillance Element & Theme \\
\hline \multirow{4}{*}{$\begin{array}{l}\text { Factors that Influence the Recognition of } \\
\text { Medical Device-Related Incidents }\end{array}$} & Education and training \\
\hline & $\begin{array}{l}\text { Hospital staff knowledge } \\
\text { and experience }\end{array}$ \\
\hline & $\begin{array}{l}\text { Performance of medical } \\
\text { device }\end{array}$ \\
\hline & Warnings or advisories \\
\hline \multirow[t]{6}{*}{$\begin{array}{l}\text { Factors that Influence the Reporting of } \\
\text { Medical Device-Related Incidents }\end{array}$} & $\begin{array}{l}\text { Clinician communication } \\
\text { with patient }\end{array}$ \\
\hline & $\begin{array}{l}\text { Feedback on how reported } \\
\text { information is used }\end{array}$ \\
\hline & $\begin{array}{l}\text { Incentives for error } \\
\text { reporting }\end{array}$ \\
\hline & Information sharing \\
\hline & Institutional culture \\
\hline & $\begin{array}{l}\text { Reporting system and } \\
\text { process }\end{array}$ \\
\hline \multirow{7}{*}{$\begin{array}{l}\text { Factors that Influence the Resolution of } \\
\text { Medical Device-Related Incidents }\end{array}$} & Education and training \\
\hline & $\begin{array}{l}\text { Feedback on how reported } \\
\text { information is used }\end{array}$ \\
\hline & $\begin{array}{l}\text { Future use of medical } \\
\text { device }\end{array}$ \\
\hline & Information sharing \\
\hline & Institutional culture \\
\hline & $\begin{array}{l}\text { Medical device procurement } \\
\text { process }\end{array}$ \\
\hline & Preventive actions \\
\hline \multirow{13}{*}{$\begin{array}{l}\text { Barriers to the Recognition, Reporting and } \\
\text { Resolution of Medical Device-Related } \\
\text { Incidents }\end{array}$} & Conflicts of interest \\
\hline & Education and training \\
\hline & Error reporting compliance \\
\hline & $\begin{array}{l}\text { Feedback on how reported } \\
\text { information is used }\end{array}$ \\
\hline & $\begin{array}{l}\text { Hospital staff knowledge } \\
\text { and experience }\end{array}$ \\
\hline & Impact on patient care \\
\hline & Liability \\
\hline & $\begin{array}{l}\text { Medical device procurement } \\
\text { process }\end{array}$ \\
\hline & $\begin{array}{l}\text { Performance of medical } \\
\text { device }\end{array}$ \\
\hline & $\begin{array}{l}\text { Personal attitude of health } \\
\text { care professional }\end{array}$ \\
\hline & Problem solving \\
\hline & Professional culture \\
\hline & $\begin{array}{l}\text { Response from } \\
\text { manufacturers }\end{array}$ \\
\hline \multirow{4}{*}{$\begin{array}{l}\text { Interventions or Strategies to Improve the } \\
\text { Recognition, Reporting and Resolution of } \\
\text { Medical Device-Related Incidents }\end{array}$} & Education and training \\
\hline & Institutional culture \\
\hline & Professional culture \\
\hline & $\begin{array}{l}\text { Reporting system and } \\
\text { process }\end{array}$ \\
\hline
\end{tabular}

near misses, or anything that could lead to an error. They also observed that hospitals have become less punitive over time when an incident was reported and adopted more of a learning culture instead, where incidents were used as an education opportunity for the hospital staff. According to one physician, the quality of care committee in his hospital oversaw operating room (OR) issues and insisted that incident reports be submitted regularly.

Based on the interview responses, physicians, nurse managers or hospital inventory suppliers reported device failures and malfunctions directly to the manufacturer and, in some instances, returned defective parts to the company. The respondents, however, did not indicate the threshold of safety adverse effects for which the defective parts were returned to the manufacturer. Furthermore, hospitals were eligible for a reimbursement from the manufacturer if a device must be replaced due to a malfunction or breakage. In instanceswhere a physician was unaware of the reporting process, he or she asked the RN to report the incident on his or her behalf. The biomedical engineering department also would receive a report about the device incident. If a hospital error reporting system existed, device incidents, malfunctions or near misses typically were documented in an operative or incident report, error database, or a patient's chart.

An internal registry was used to track the results of vascular surgeries in one hospital, but this type of system was not available in all clinical areas, including interventional radiology. The same hospital also participated in a global vascular qualitative initiative that allowed vascular surgeons to submit data about perioperative patient and the procedure performed. Another surgeon stated that a system is in place within his hospital, where malfunctioned devices were registered for tracking purposes, along with the actual malfunction and surgical team involved. The respondent did not elaborate on how the data was used by the hospital. In one hospital, RNs reported problems or complications with patient care in a patient safety and learning system.

\section{Factors that influence resolution of medical device-related incidents}

Themes related to the resolution of incidents included education and training, future use of medical device, information sharing, institutional culture, medical device procurement process, and preventive actions. It was common practice for physicians to organize morbidity and mortality rounds on a regular basis in their department and to discuss medical device-related incidents as part of the quality improvement process for patient care. Physicians also participated in interdepartmental formal rounds to learn from colleagues and to discuss potential solutions to the problem. Some interviewees felt that adequate training by the hospital and manufacturers on the use 
of a current or new medical device for new staff and continuous education on how to operate a medical device would help the end-user to understand the medical device functionality and to recognize potential errors and malfunctions associated when used on a patient. Training on error reporting systems and processes also would benefit the hospital staff.

For malfunctioning devices, one respondent stated that the hospital returned equipment to manufacturer and requested a refund. The most common decision among respondents related to a device malfunction was its disinvestment or discontinued use in clinical procedures.

Several respondents felt that it was important for their hospital purchasing department to review the contractual obligations with the manufacturers and incorporate the appropriate clauses to help ensure that they addressed adequately any performance issues observed with their medical devices. Also, device incidents and malfunctions reported by the hospital staff were used to negotiate terms and agreements with manufacturers for future contracts. One orthopaedic surgeon revealed that his hospital checked the medical devices and kept records of when they were last tested.

As well, respondents described prevention actions that were in place in their department. They included safety checklists to mitigate and manage incidents, planning for the off-label use of a device in a specific case in a multidisciplinary team and how to address a similar case in the future, and a clinical review of manufacturer instructions to determine they were appropriate.

\section{Barriers to recognition, reporting and resolution of medical device-related incidents}

Themes related to barriers include conflicts of interest, education and training, error reporting compliance, feedback on how reported information is used, hospital staff knowledge and experience, impact on patient care, liability, medical device procurement process, performance of medical device, personal attitude of health care professional, problem solving, professional culture, and response from manufacturers. A lack of awareness of implications on patient care if not reporting errors and how to report a medical device-related incident or malfunction, among hospital staff were common barriers cited by numerous respondents. One RN and one cardiac surgeon from the same institution were discouraged by the lack of follow-up from the hospital when an incident or a malfunction was reported and felt that nothing happened. Conversely, it was reported that, in one instance, the manufacturer used the information reported by the hospital to improve the design and functionality of the medical device.

A high turnover with some medical devices rendered it a challenge for staff members to gain experience with them and, thus, understand the nuances of their performance. One radiologist found it was demanding at times to interpret imaging information performed in another institution and indicated that clinicians who transferred patients with complex profiles to a tertiary care hospital did not always have the capacity to recognize medical device-related problems. Another surgeon suggested that the success of a clinical procedure also depended on the proper set up of instruments by the RN. If a medical device incident was infrequent, some hospital staff members were less aware of the error report system and, subsequently, did not report the event. A general surgeon expressed some frustration when the nursing staff in the OR had limited knowledge on the proper use of the device, so delays occurred during the clinical procedure. One RN acknowledged that the nursing staff did not always have the expertise to troubleshoot problems observed in a medical device and found it challenging to differentiate between an incident and a near miss.

Some respondents were intimidated by punishment and potential legal consequences and feared a loss of credentials or privileges at their institution if they reported a device incident. Others also felt that error reporting to the hospital was complicated and time-consuming. Both RNs and physicians were concerned about their professional reputation if they reported an error. Several respondents did not think that there were any barriers to recognizing, reporting and resolving medical device-related incidents in their area of practice.

In terms of resolving a device incident, challenges arose in troubleshooting a unique circumstance in real-time even when the manufacturer representative was present in the OR in some instances. It is unclear if manufacturers frequently had difficulties troubleshooting device incidents. Hospital staff did not always want to discuss each other's complications with the use of medical devices, but some felt that they had a professional obligation to report a device incident or malfunction to the hospital. One RN suspected that there was a lack of compliance among her colleagues in conducting independent checks in the programming of the medical equipment. Both physicians and RNs expressed their dissatisfaction with manufacturers or distributors when they provided limited information about the device incident or malfunction reported by the hospital. An inadequate response, therefore, hindered the hospital staff from resolving the problem.

\section{Initiatives or suggestions to improve recognition, reporting and resolution of medical device-related incidents}

Themes that emerged from their responses include education and training, institutional and professional cultures, and reporting system and process. Hospitals must ensure that their staff receives adequate training on the use of new medical devices and existing error reporting systems 
and how to report device incidents and malfunctions to ensure consistency in clinical practice.

Although post-market surveillance systems for devices, such as the FDA MAUDE database, exist and error reports are available in the public domain, one physician felt that there should be increased collaboration among hospital facilities to track the performance of medical devices. Similar collaborations existed for the purchase of medical supplies and equipment, so he wondered why a similar one had not been established yet for medical device-related incidents and malfunctions. The respondent did not comment on existing post-market surveillance systems, so his thoughts on their effectiveness to improve the safety of medical devices are uncertain. One respondent observed an expansion of processes and practices over time, such as morbidity and mortality, from vascular surgery to interventional radiology. One RN suggested that RNs play a more active role in refusing to use a medical device that did not perform as intended and allow frontline staff, who will use the medical device in practice, to test and understand how it works. As well, the same respondent felt that RNs should be more empowered and be allowed to refuse to use defective medical equipment.

A surgeon indicated that a provincial-wide vascular database would be implemented in his hospital. This database would capture all vascular procedures prospectively but is not limited to device incidents. A couple of physicians suggested setting up an infrastructure to facilitate the automation of reporting medical device-related incidents and malfunctions. This approach would reduce the occurrence of device problems going unnoticed. Some respondents felt that hospitals should collaborate to develop and adopt a global registry to capture the performance of medical devices used in their institutions. Such a registry would help to determine more accurately the denominator in the number of procedures associated with specific devices. Several RNs and physicians were unaware of any initiatives underway in their hospitals for device incident recognition, reporting and resolution.

\section{Interpretation}

Even though the literature identified the performance of a health technology as a factor that influenced the recognition of incidents, numerous respondents attributed the recognition of incidents to devices not operating as intended and to the hospital staff's knowledge and professional experience on both medical device performance and clinical manifestations of patients. When a device incident had occurred, patients and their families always were notified, manufacturers were contacted, and hospital incident forms were completed. If the device incident was deemed to be serious according to the physician's judgment, then the regulators also were advised. Based on the physician's response, it was a challenge to discern if the error reporting to various parties was consistent. Conversely, errors not deemed to be serious by the physician or nurse or near misses were not always reported to the hospital. Several participants felt that the manufacturer's response to a device incident provided insufficient information to resolve the problem, and they were reluctant or averse to reuse the medical device in future clinical procedures. Some, therefore, recommended that the hospital purchasing department incorporate appropriate clauses in new or renewed contracts with the manufacturer. The telephone interviews also revealed that physicians discontinued using a specific device if it malfunctioned and the problem was not resolved and the risk, therefore, of a similar incident reoccurring continued to exist. Should an alternative therapy not be available, then risk mitigation strategies developed by the hospital department were planned for future clinical procedures.

Respondents indicated that a medical device surveillance program that incorporated a medical device registry, increased education and training and an open communication and feedback strategy with hospital administrators and managers to encourage information sharing and increase incident reporting would contribute to increased patient safety in a hospital facility. More specifically, education and training programs on the incident recognition and appropriate use of new medical devices to enhance their performance on patient care; greater awareness on error reporting and its significance; increased feedback from management to staff about reported errors; and modifications to the medical device purchase process to increase the manufacturer's accountability would ameliorate medical device surveillance in a hospital facility.

\section{Implications}

The results of our telephone interviews and a systematic review were used to propose a conceptual framework on medical device surveillance in hospitals (Fig. 1). [7] In addition to education and training, an open communication and feedback strategy, and an adverse medical device database/registry, the proposed framework would integrate medical devices with information systems to identify, track, and manage their location. This framework would capture and evaluate the appropriate resolutions to help lower the risk of incidents and adverse events associated with medical device use in hospitalized patients [15]. Prior to the design and development of a medical device surveillance system, a comparison and an assessment of the safety mechanisms in place in other technology-related sectors, such as aviation and machinery and equipment, is warranted. The feasibility and appropriateness of these mechanisms for a device surveillance system in a hospital facility also would be investigated. 


\section{CONCEPTUAL FRAMEWORK FOR MEDICAL DEVICE SURVEILLANCE IN A HOSPITAL CONTEXT}

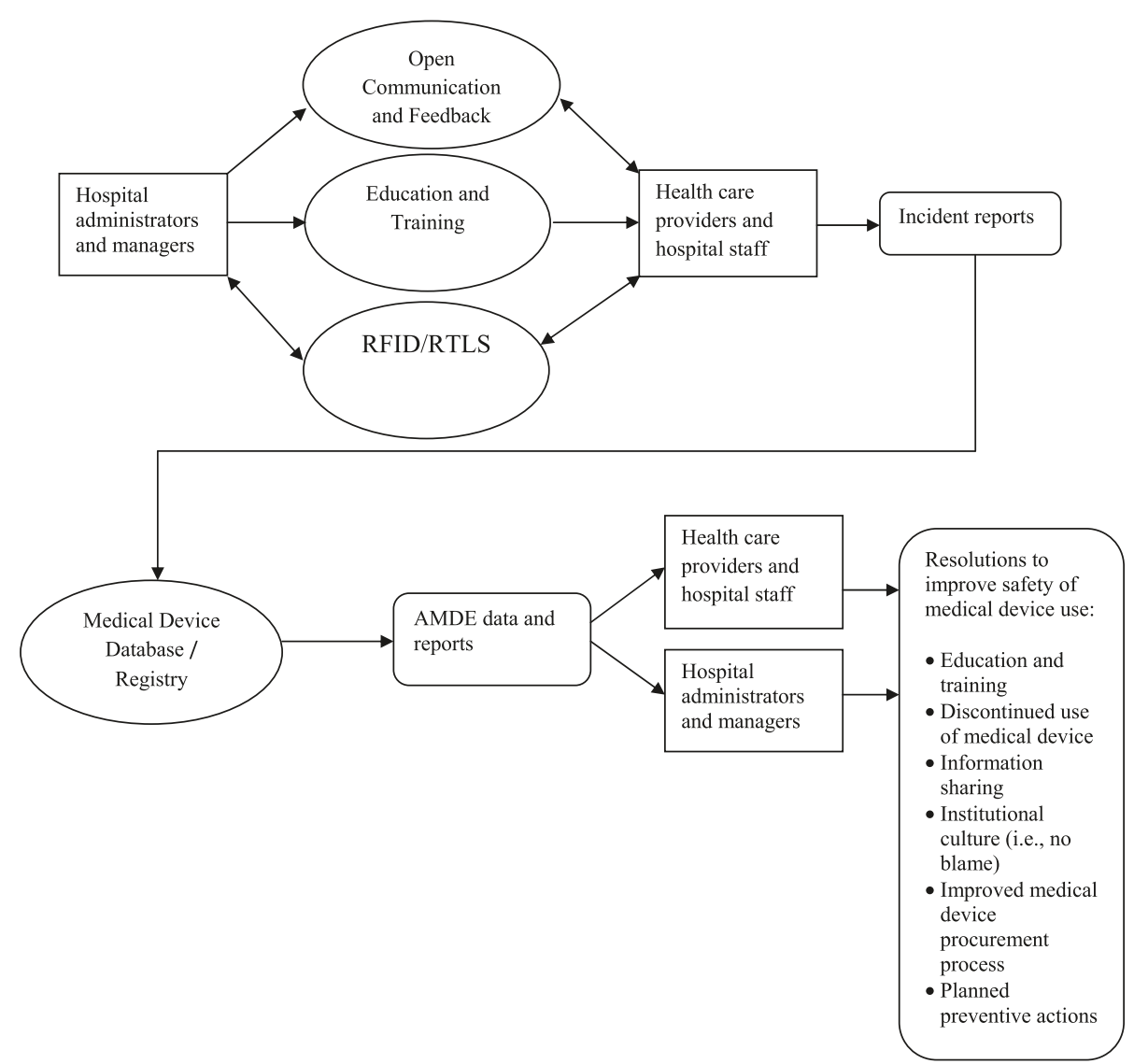

Fig. $1 \mathrm{AMDE}=$ Adverse medical device event; RFID/RTLS=Radio frequency identifier/real-time location systems

\section{Discussion}

Sixteen interviews with physicians and RNs were conducted to explore factors that influence recognition, reporting and resolution of device incidents, to identify barriers to, and to suggest areas for improvement for medical device safety surveillance. Although previous studies developed a framework on incident reporting to improve patient safety in a hospital context $[4,16,17]$, this is the first study to collect data on factors that influence the recognition, reporting and resolution of medical device incidents and improvement strategies. The findings of the interviews were used to design and develop a conceptual framework to improve the safety of medical devices in hospitals (Fig. 1). Responses related to barriers to reporting and suggestions to improve the surveillance of incidents were aligned with the results of a previous systematic review [18].

To help hospital staff learn from a medical devicerelated incident, Amoore and Ingram developed an anonymous feedback framework that included a description of the incident, its cause, lessons learnt, corrective actions taken, and interventions to help prevent errors [19].
Although not specific to a hospital setting, Maisel argued for a staged notification if a patient's medical device was recalled, where physicians were notified first by the manufacturer. In turn, they notified the patients. This approach facilitates shared decision making between the physician and patient on the appropriate course of action. Since the use of medical devices in clinical practice continues to expand, rigorous monitoring for medical device performance, the introduction of unique device identifiers on devices to improve their traceability in the United States and Europe, greater awareness of device malfunctions and demand for increased reliability of medical devices is warranted [20, 21]. A systematic review on case studies that illustrate disinvestment and resource allocation decision making process revealed that six of the 14 studies examined the safety and adverse event evidence of the health care technologies and services proposed for disinvestment. It was unclear, however, the impact of the evidence during the decision process [22].

Interpretation and application of the findings may be influenced by several limitations. Responses from the 
telephone interviews may not be transferable to community care hospitals or other health care settings as tertiary care institutions that differ in terms of clinical procedures performed, medical devices used and hospital policies, processes and procedures. Purposive sampling was employed, so participants were not randomly selected but were invited to participate to provide diverse perspectives according to years of practice and specialty areas. There was a potential for responder bias, where physicians and RNs, who were more interested in medical device-related incidents compared with their colleagues, agreed to be interviewed, influencing the responses. For example, we attempted to recruit more RNs from each hospital, but they indicated that they were unable to participate due to their schedules. We, however, ensured that at least one RN in the OR and ICU participated in our study and our analysis of their responses suggested similarities in their perspectives and experiences in medical device incidents in hospitals. Moreover, the authors were unable to ascertain if the devices incidents occurred due to the device malfunction, user error or insufficient knowledge on their proper use, as well as the severity of device incidents. As telephone interviews were conducted for practical reasons, the interviewer was unable to see interviewee's physical gestures or nuances that can been seen in person. Instead, she relied on the interviewees' audio cues.

Recent initiatives are developing innovative study designs to improve the reliability of premarket assessment on the use of medical devices in "real-world" settings $[23,24]$. New paradigms propose hierarchical modeling that combines premarket and postmarket data to illustrate the effectiveness and safety of a medical device throughout the total product lifecycle [25]. Continued research in this area can be used to inform recommendations that strengthen post-market device surveillance, as well as integration with pre-market data. The telephone interviews in the our study illustrate some of the perceptions of physicians and nurses related the recognition, reporting and resolution of medical device-related incidents, further investigation is required to understand the causes and rationale behind their thought processes and behaviours. For instance, follow-up interviews would provide some insights on the physician and nurse perceptions of devices the risk they pose to their patients, as well as elucidate why physicians and RNs are concerned about their professional reputation if it is their obligation to deliver health care to and ensure the safety of their patients. Interviews with physicians with other specializations, hospital administration staff, the biomedical engineering department and manufacturers would reveal additional information on factors that influence the recognition, reporting and resolution of device incidents. Furthermore, an exploration of exemplar Canadian and international medical device surveillance systems and their features, and their application in a hospital can lead to the development of a minimum data set for incident reporting. These developments merit further investigation as they will help to ameliorate patient care outcomes and health care delivery. Future research can further investigate the device malfunctions cited by the respondents and compare them to reports of recalls to assess their prevalence and severity in clinical practice. In addition, follow-up interviews to review how device malfunctions were handled after they reported errors to the hospital and compare the results to their written policies and procedures that explicitly states the principals and course of actions merits an investigation. The findings of the aforementioned research priorities areas also would contribute to the design and development of an effective medical device hospital surveillance system, including the appropriate error reporting system, education and training, and communication strategies.

\section{Conclusions}

Sixteen interviews were conducted among physicians and RNs in two teaching hospitals. The hospital staff's knowledge and experience, as well as the patient's clinical characteristics and device performance were important factors in incident recognition. Incident severity, awareness and ease of use of reporting systems and processes, as well as organizational culture and personal attitudes and perceptions of responses contributed to the frequency of reporting device incidents. The discontinued use of a medical device or equipment was the most common resolution to prevent the reoccurrence of a similar error. Suggested strategies for improvement involved education and training, institutional and professional cultures, and improved reporting systems and processes. The results of this qualitative study has the potential to provide some initial steps to the design and development of a hospital medical device surveillance system that would help to improve the patient safety and health care delivery.

\section{Additional files}

Additional file 1: Interview guide.

Additional file 2: Table S1. Thematic summary of interview responses with exemplar quotes.

Competing interests

The authors declare that they have no competing interests.

\section{Authors' contributions}

JP led the qualitative study and preparation of the manuscript. AG participated in the analysis of the interview responses and contributed and reviewed the draft versions of the manuscript. TJC contributed to and reviewed the draft versions of the manuscript. All authors read and approved the final manuscript. 


\section{Acknowledgements}

This work has been completed in partial fulfillment of the requirements for Julie Polisena's PhD degree in Epidemiology at the University of Ottawa. Julie Polisena is funded by the University of Ottawa Admission Scholarship. The authors would like to acknowledge Kathleen Power for her transcription services.

\section{Author details}

${ }^{1}$ Canadian Agency for Drugs and Technologies in Health, 600-865 Carling Avenue, Ottawa, ON K1S 5S8, Canada. ${ }^{2}$ Department of Epidemiology and Community Medicine, Faculty of Medicine, University of Ottawa, 451 Smyth Road, Ottawa, ON K1H 8 M5, Canada. ${ }^{3}$ Toronto General Research Institute, University Health Network, 200 Elizabeth Street, Toronto, ON M5G 2C4, Canada.

Received: 14 May 2014 Accepted: 20 May 2015

Published online: 06 June 2015

\section{References}

1. Maisel WH. Medical device regulation: an introduction for the practicing physician. Ann Intern Med. 2004;140:296-302.

2. Balka E, Doyle-Waters M, Lecznarowicz D, FitzGerald JM. Technology, governance and patient safety: systems issues in technology and patient safety. Int J Med Inform. 2007;76 Suppl 1:S35-47.

3. Baker GR, Norton PG, Flintoft V, Blais R, Brown A, Cox J, et al. The Canadian Adverse Events Study: the incidence of adverse events among hospital patients in Canada. CMAJ. 2004;170:1678-86.

4. Lawton R, McEachan RR, Giles SJ, Sirriyeh R, Watt IS, Wright J. Development of an evidence-based framework of factors contributing to patient safety incidents in hospital settings: a systematic review. BMJ Qual Saf. 2012;21:369-80.

5. Sievanen $\mathrm{H}$. User reporting of medical device related incidents. Med Device Technol. 2003:14:26-9.

6. Pfeiffer $Y$, Manser T, Wehner T. Conceptualising barriers to incident reporting: a psychological framework. Qual Saf Health Care. 2010;19, e60.

7. Polisena J, Gagliardi A, Urbach D, Clifford T, Fiander M. Factors that influence the recognition, reporting and resolution of medical devicerelated incidents: a systematic review, Systematic Reviews. 2015;4:37.

8. Strauss A, Corbin J. Basics of qualitative research: grounded theory procedures and techniques. Newbury Park (CA): Sage Publications; 1998

9. Butterfield LD, Borgen WA, Amundson NE, Maglio AST. Fifty years of the critical incident technique: 1954-2004 and beyond. Qualitative Research. 2005:5:475-97.

10. Bowen GA. Naturalistic inquiry and the saturation concept: a research note. Qualitative Research. 2008:8:137-52.

11. Sandelowski M. Theory unmasked: the uses and guises of theory in qualitative research. Res Nurs Health. 1993;16:213-8.

12. Cooper JB, Newbower RS, Kitz RJ. An analysis of major errors and equipment failures in anesthesia management: considerations for prevention and detection. Anesthesiology. 1984;60:34-42.

13. Auerbach CF, Silverstein LB. Qualitative data: an introduction to coding and analysis. New York: New York University Press; 2003.

14. Pope C, Ziebland S, Mays N. Qualitative research in health care. Analysing qualitative data. BMJ. 2000;320:114-6.

15. Polisena J, Jutai J, Chreyh R. A proposed framework to improve the safety of medical devices in a Canadian hospital context. Medical devices: research and evidence. Medical Devices: Research and Evidence 2014.

16. MaRS Discovery District. How medical devices are approved in the US MaRS Discovery District. 2010. Toronto (ON), MaRS. 11-11-2013.

17. Kaldjian LC, Jones EW, Rosenthal GE, Tripp-Reimer T, Hillis SL. An empirically derived taxonomy of factors affecting physicians' willingness to disclose medical errors. J Gen Intern Med. 2006;21:942-8.

18. Segal JB, Bridges JF, Chang HY, Chang E, Nassery N, Weiner J, et al Identifying possible indicators of systematic overuse of health care procedures with claims data. Med Care. 2014:52:157-63.

19. Amoore J, Ingram P. Quality improvement report: Learning from adverse incidents involving medical devices. BMJ. 2002;325:272-5.

20. Sorenson C, Drummond M. Improving medical device regulation: the United States and Europe in perspective. Milbank Q. 2014;92:114-50.

21. Maisel WH. Safety issues involving medical devices: implications of recent implantable cardioverter-defibrillator malfunctions. Journal of the American Medical Association. 2005;294:955-8.
22. Polisena J, Clifford T, Elshaug AG, Mitton C, Russell E, Skidmore B. Case studies that illustrate disinvestment and resource allocation decision-making processes in health care: a systematic review. Int J Technol Assess Health Care. 2013:29:174-84

23. MaRS Discovery District. New partnership to establish Ontario as premier site in pre-market evaluation of medical technologies [news release]. MaRS Discovery District. 12-6-2011. Toronto (ON), MaRS. 1-2-2014.

24. U.S. Food and Drug Administration. Medical Device Epidemiology Network (MDEpiNET). U.S. Food and Drug Administration. 4-22-2014. Silver Spring (MD), U.S. Food and Drug Administration. 1-2-2014.

25. Normand SL, Marinac-Dabic D, Sedrakyan A, Kaczmarek R. Rethinking analytical strategies for surveillance of medical devices: the case of hip arthroplasty. Med Care. 2010:48:S58-67.

\section{Submit your next manuscript to BioMed Central and take full advantage of:}

- Convenient online submission

- Thorough peer review

- No space constraints or color figure charges

- Immediate publication on acceptance

- Inclusion in PubMed, CAS, Scopus and Google Scholar

- Research which is freely available for redistribution

Submit your manuscript at www.biomedcentral.com/submit 